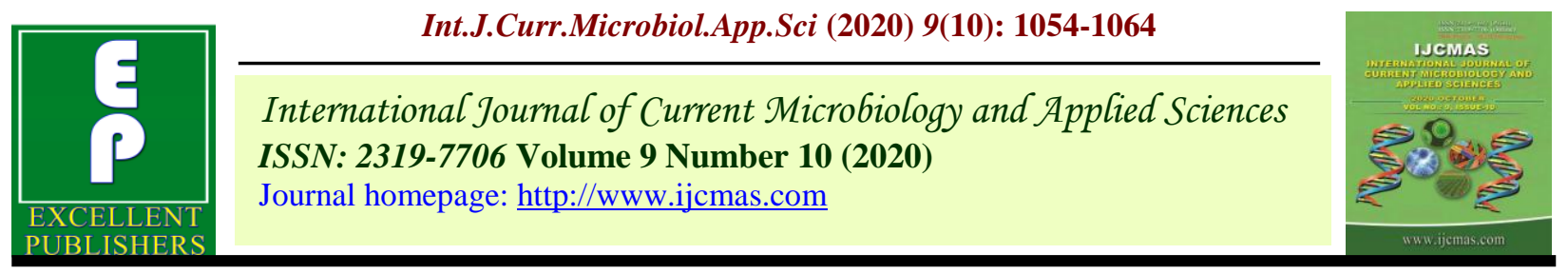

Original Research Article

https://doi.org/10.20546/ijcmas.2020.910.126

\title{
Heterosis and Combining Ability Studies in Maize (Zea mays L.) for Drought Tolerance, TLB Disease Resistance and Productivity in Northern Dry Tract of Karnataka
}

\author{
Meghashri S. Patil*, B. N. Motagi and R. M. Kachapur \\ Department of Genetics and Plant Breeding, College of Agriculture, Vijayapur, University of \\ Agricultural Sciences, Dharwad, Karnataka, India \\ *Corresponding author
}

Keywords

Linextester,

Standard heterosis,

Combining ability,

TLB, Drought

Article Info

Accepted:

10 September 2020

Available Online:

10 October 2020

\section{A B S T R A C T}

Maize being a cross pollinated crop has a higher yield potential compared to other cereals. In the present investigation, 30 full season hybrids (fifteen lines crossed with two testers in LinexTester) were evaluated for their productivity, drought tolerance and turcicum leaf blight (TLB) disease resistance in the dry tract of Northern Karnataka. The line $\times$ tester analysis was carried out to determine the standard heterosis and combining ability in these hybrids with five checks namely Bio 9544, NK-6240 (National checks), CMH08-282 (Zonal check), GPMH-1101 and BRMH-1 (Local checks) under rainfed conditions during kharif 2018 at the Regional Agricultural Research Station, Vijayapur. Among these hybrids, GHVJP5 (GPM-26×CML-451) was found to be better performing for most of the traits followed by GHVJP23 (GPM-686×CML-451); the hybrid GHVJP13 (GPM$225 \times$ CML451) was found to be drought tolerant; GHVJP5 and GHVJP12 (GPM119×CM111) were found to be resistant to TLB and GHVJP14 (GPM-225×CM111) and GHVJP5 were found to be early maturing. These hybrids can be further tested for their proficiency. Among parents, the lines GPM26 and GPM686 were found to be good general combiners for productivity traits. These can be used as parents in the development of superior hybrids.

\section{Introduction}

Maize (Zea mays L.) is a versatile crop grown over a range of agro climatic zones and is an important food and forage crop with abundant natural diversity. In India, it is cultivated in 9.6 million hectares area with 27.15 million tonnes annual production and 2.83 tonnes per hectare productivity (Anon. 2018). It is one of the most important Kharif cereal crops of Karnataka and is cultivated in 13.7 lakh hectares with 33.14 lakh tonnes production and $2419 \mathrm{~kg}$ per hectare productivity (Anon. 2017).

Maize is a highly cross-pollinated crop and scope for exploitation of hybrid-vigor depends on direction, magnitude of heterosis and type of gene action involved. The exploitation of heterosis is possible only when parents involved in crosses differ in their combining ability. In any hybridization 
program, recognition of best combination of two/more parental genotypes to maximize variance within related breeding populations, and as a result the chance of recognizing superior transgressive segregants in the segregating populations are the most critical challenges to plant breeders.

Combining ability studies provide information on the genetic mechanisms controlling inheritance of quantitative traits and enable breeders to select suitable parents for further improvement or use in hybrid breeding for commercial purposes.

One of the main deterrents to high grain yield in maize is its susceptibility to several diseases. Of 112 diseases of maize reported so far from different parts of the globe, 65 are known to occur in India and in zone 3 of Karnataka, TLB (Exerohilum turcicum) and MLB (Bipolaris maydis) are found to be of common occurrence. TLB is one of the most important and widespread fungal disease infecting leaves prior to initiation of flowering and later spreading to cover entire leaves thereby affecting photosynthesis resulting in the severe reduction in grain yield. Hence resistance to TLB is one of the important criteria in development of new hybrid combinations.

Northern dry zone of Karnataka (zone-3) is where new areas of maize is expanding due to increase in command area. Hence in the present study an attempt is made to evaluate the public bred hybrids to know their combining ability, heterosis, resistance to TLB, drought tolerance and productivity traits.

\section{Materials and Methods}

The experiment was conducted at Regional Agricultural Research Station, Vijayapura in kharif 2018 located at $16^{\circ} 49^{\prime} \mathrm{N}$ latitude, $75^{\circ}$
43' E longitude and $593 \mathrm{~m}$ above mean sea level with a mean annual rainfall of $590 \mathrm{~mm}$. The rainfall during 2018 was $466.3 \mathrm{~mm}$. June and September were the wettest months during 2018 as compared to other months. During the year 2018, maximum monthly mean temperature was the lowest in December $\left(29.70^{\circ} \mathrm{C}\right)$, while highest was in May $\left(39.20^{\circ} \mathrm{C}\right)$. Minimum monthly mean temperature was the highest in May $\left(23.40^{\circ} \mathrm{C}\right)$ and the lowest in January $\left(13.0^{\circ} \mathrm{C}\right)$.

Thirty hybrids developed by crossing 15 lines and two testers in linextester mating design (Kempthorne 1957) were collected from AICMIP, College of Agriculture, UAS, Dharwad. These crosses were attempted in Rabi 2017-18 at UAS, Dharwad.

Lines: GPM-04, GPM-07, GPM-26, GPM-52, GPM-114, GPM-119, GPM-225, GPM-255, GPM-628, GPM-668, GPM-676, GPM-686, GPM-735, GPM-751 and GPM-755.

Testers: CML-451 and CM-111.

Checks: Bio 9544, NK-6240 (National checks), CMH 08-282 (Zonal Check), GPMH-1101 and BRMH-1 (local Checks).

The randomised complete block design with three replications was followed to evaluate the experimental hybrids. Each plot consisted of two rows of three meter length with inter and intra-row spacing of $60 \mathrm{~cm}$ and $20 \mathrm{~cm}$, respectively and all the package of practice was followed for normal cultivation of crop. From each entry/replication, five random, competitive plants were tagged and numbered in the middle of row to observe yield and other parameters. The observations were recorded as follows. Days to $50 \%$ anthesis and days to $50 \%$ silking were recorded from date of sowing on per plot basis. Anthesissilking interval was determined by the difference between days to $50 \%$ anthesis and 
$50 \%$ silking. The plant height was recorded in centimeter from the ground level to the base of the tassel and the ear height was recorded in centimeter from the ground level to the base of the uppermost ear in five selected plants in each genotype at maturity. The cob length was measured in centimeter from the base to the tip of the cob after harvest in five selected plants in each genotype. For measuring cob girth, ordinary string or thread was wrapped in the middle portion of the ear and the length of thread was measured in centimeter and noted in terms of perimeter. Then the perimeter was converted to diameter by dividing the values by 3.14 .

Days to maturity, kernel row number, kernel per row, cob weight, grain weight, test weight, fodder yield and grain yield were measured as usual. The relative chlorophyll content was measured for the third leaf from the top at 60 days after sowing on five randomly selected plants in each genotype using SPAD chlorophyll meter. For measuring relative water content, fully expanded and mature leaves were selected from five randomly selected plants at 60 DAS and their fresh weight was noted down. Then the leaf samples were immersed in water for $8 \mathrm{hrs}$ and then the turgid weight of the samples was measured. These samples were further dried in oven at $60^{\circ} \mathrm{C}$ and the dry weight was determined. The relative water content was then calculated using the following formula.

RWC $(\%)=\left(\frac{\text { Fresh weight }- \text { Dry weight }}{\text { Turgid weight }- \text { Dry weight }}\right) \times 100$

Disease screening for TLB was done under natural conditions by following one to five disease rating scale given by Payak and Sharma (1983) at the respective stages of the crop growth. The data thus obtained was analysed statistically in TNAUSTAT software.

\section{Results and Discussion}

\section{Analysis of variance}

The analysis of variance (Table 1) for different traits revealed that, there was significant variation due to lines for all the traits studied which indicated that the lines were genetically diverse among themselves and the variation due to testers was significant for most of the traits except PH, CL, CG and SP. However, the mean sum of squares due to testers was higher than that due lines for majority of traits indicating that the testers were more genetically diverse than lines. Since there was greater diversity in the parents we can expect the crosses between them to be heterotic. There was significant variation among the crosses for all the traits studied except for plant height.

\section{Combining ability}

The identification of good general combiners for different traits and partitioning of the parents into good and poor combiners helps us in identifying and utilizing the better parents in hybridization programmes. Also identification of hybrids with good SCA helps in exploiting heterosis from such crosses.

For early maturity, the lines GPM-225, GPM04 and tester CM-111 were found to be good general combiners (Table 2a) as they had significant negative GCA effects for DFA, DFS and DM. Thus these lines can be used as parents in the development of hybrids suitable for early maturing. The hybrids GHVJP1 (GPM-04×CML-451), GHVJP5 (GPM26×CML-451) and GHVJP14 (GPM$225 \times \mathrm{CM}-111)$ were found to have significant negative sca (Table 3a) for days to fifty percent flowering indicating their earliness and this may be due to the narrow sense heritability of the trait (Panda et al., 2017). These hybrids showing early maturity also 
had good per se performance for yield and related traits and hence can be suitable for cultivation in drought prone areas like zone 3 so that they can escape drought to considerable extent. These plants can also be used in mixed or inter cropping systems as well. Similar results were obtained by Dubey et al., (2001).

For drought tolerance the traits like ASI, RWC and SPAD were taken into consideration. The line GPM-686 was found be good general combiner for RWC and SPAD while GPM-668 was found to be good general combiner for all these traits (Table 2a) and hence can be used as source for drought tolerance. The hybrid GHVJP13 (GPM$225 \times$ CML-451) which is a cross between a good $\times$ poor combiner had significant sca effects in positive direction for relative water content (Table 3a) and also had higher yields. This indicated that the high gca of parent doesn't always assure significant sca among hybrids. This hybrid can be considered as drought tolerant and can be subjected to further evaluation in multi locations.

For TLB resistance, the lines GPM-26, GPM119, GPM-751 and tester CM-111 were found to be good general combiners (Table $2 \mathrm{a}$ ) and can be used as source of resistance. Among the hybrids, GHVJP5 (GPM-26×CML-451) and GHVJP12 (GPM-119×CM111) were found to be resistant to TLB. These GCA estimates indicated the possibility to select inbred lines that can be combined to generate hybrids that are resistant to the disease (Ejigu et al., 2017).

With respect to productivity traits, the line GPM-26 was found to good general combiner for CL, CW, GW, SP and TW; the line GPM686 was found to be good general combiner for CL, CG, KPR and GY (Table 2b); the lines GPM-735 and GPM-628 were found to be good general combiners for KRN (Table 2b) and the line GPM-119 was found to be good general combiner for FY (Table 2b). Among testers, CML-451 was found to be good general combiner for KRN and TW; CM-111 was found to be good general combiner for GY (Table 2b). As these traits indirectly contribute to increased yields these can be used as parents for developing elite hybrids.

The hybrids GHVJP5 (GPM-26×CML-451) and GHVJP23 (GPM-686×CML451) showed significant positive SCA for CL, KPR, CW, GW and GY (Table 3b). The hybrid GHVJP12 (GPM-119×CM111) had significant positive SCA for KPR and GY (Table $3 b$ ) and was also found to be resistant to TLB. The hybrid GHVJP13 (GPM$225 \times$ CML-451) showed significant positive SCA for KRN, GY (Table 3b) and was also found to be drought tolerant. The hybrid GHVJP25 (GPM-735×CML451) showed significant positive SCA for GY (Table 3b) with high per se values for the same. Thus these hybrids can be subjected to further evaluation for testing their proficiency.

The lines and testers were classified into good and poor general combiners, pooled gca effects for different characters was taken into consideration. This was done by assigning the value " +1 " for positive gca effects and " 0 " for negative gca effects and then the values were summed up. If the value of a line or tester had more than average value, it was considered as good combiner or else designated as poor combiner.

In the present study, the crosses involving both good general combiner (GHVJP5 and GHVJP23) as well as one good and one poor combiner (GHVJP12, GHVJP13 and GHVJP25) showed high SCA effects which are due to additivexadditive and additivexdominant gene action and confirmed by earlier reports of Amin et al., (2014). 
Those promising crosses that were due to additivexadditive gene action were identified as overall high general combiners and could be utilized for development of either the synthetic varieties or an elite breeding population by allowing greater recombination among them to achieve new genetic recombination and then subjecting the resultant population to recurrent selection (Izhar and Chakraborty, 2013).

Table.1 ANOVA for line $\mathrm{x}$ tester for yield traits

\begin{tabular}{|c|c|c|c|c|c|c|c|c|c|c|c|}
\hline \multirow{2}{*}{ Source } & \multicolumn{10}{|c|}{ MSS } \\
\hline & df & PH & CL & CG & KRN & KPR & CW & GW & SP & TW & GY \\
\hline Replication & 2 & 564.49 & 8.34 & 0.91 & 1.99 & 2.33 & 2914.99 & 1051.87 & 29.73 & 16.60 & 0.53 \\
\hline Genotypes & 34 & $282.64^{*}$ & $6.59^{* *}$ & $1.57^{* *}$ & $1.84^{* *}$ & $24.53^{*}$ & $3130.35^{* *}$ & $2243.06^{* *}$ & $18.62^{*}$ & $29.35^{* *}$ & $0.69^{* *}$ \\
\hline Crosses & 29 & 287.40 & $7.29^{* *}$ & $1.35^{* *}$ & $1.84^{* *}$ & $25.01^{*}$ & $3549.30^{* *}$ & $2527.22^{* *}$ & $17.28^{*}$ & $31.12^{* *}$ & $0.77^{* *}$ \\
\hline Lines & 14 & $382.71^{*}$ & $10.94^{* *}$ & $1.29^{*}$ & $1.58^{* *}$ & $27.21^{*}$ & $4503.66^{* *}$ & $3321.51^{* *}$ & $24.54^{* *}$ & $49.06^{* *}$ & $0.98^{* *}$ \\
\hline Testers & 1 & 5.98 & 7.74 & 0.80 & $6.83^{* *}$ & $86.83^{*}$ & $4557.09^{*}$ & $2048.91^{*}$ & 19.62 & $100.28^{* *}$ & $2.06^{* *}$ \\
\hline Line x Tester & 14 & 212.18 & 3.60 & $1.44^{* *}$ & $1.75^{* *}$ & 18.39 & $2522.97 * *$ & $1767.11^{* *}$ & 9.87 & 8.24 & $0.46^{* *}$ \\
\hline Error & 68 & 200.51 & 2.12 & 0.58 & 0.56 & 12.38 & 801.33 & 492.71 & 8.61 & 6.95 & 0.16 \\
\hline CV (\%) & & 9.03 & 8.60 & 5.53 & 5.48 & 10.62 & 16.30 & 16.49 & 3.91 & 9.69 & 12.56 \\
\hline CD at 5\% & & 22.97 & 2.46 & 1.32 & 1.25 & 5.67 & 46.17 & 36.16 & 4.89 & 4.16 & 0.64 \\
\hline CD at 1\% & & 30.51 & 3.27 & 1.75 & 1.66 & 7.53 & 61.32 & 48.04 & 6.49 & 5.53 & 0.85 \\
\hline
\end{tabular}

df: degrees of freedom, $*$ and $* *$ : Significance at $5 \%$ and $1 \%$ probability respectively

Table.2a The GCA effects of parents for different morpho-physiological traits

\begin{tabular}{|c|c|c|c|c|c|c|c|c|c|}
\hline Parents & DFA & DFS & ASI & EH & PH & SPAD & RWC & DM & TLB \\
\hline Lines & & & & & & & & & \\
\hline GPM-04 & $-1.84 * *$ & $\mathbf{- 1 . 9 9} * *$ & -0.14 & 3.31 & 6.49 & -0.03 & $4.39 * *$ & $\mathbf{- 1 . 9 9} * *$ & $0.56 * *$ \\
\hline GPM-07 & $-1.68 * *$ & $-1.49 * *$ & 0.19 & 5.14 & 8.66 & -2.47 & $1.82 * *$ & $-1.49 * *$ & 0.12 \\
\hline GPM-26 & $1.32 * *$ & $0.84 *$ & $-0.48 * *$ & $11.31 * *$ & $17.82 * *$ & -1.57 & $-1.44 * *$ & $0.84 *$ & $-0.69 * *$ \\
\hline GPM-52 & $1.49 * *$ & $2.01 * *$ & $0.52 * *$ & 4.98 & 1.16 & 0.30 & $8.26 * *$ & $2.01 * *$ & 0.19 \\
\hline GPM-114 & $2.16 * *$ & $1.84 * *$ & -0.31 & -1.52 & -8.18 & -3.03 & $4.51 * *$ & $1.84 * *$ & 0.33 \\
\hline GPM-119 & -0.51 & -0.32 & 0.19 & -4.86 & -2.18 & 3.07 & $-7.27 * *$ & -0.32 & $-0.53 * *$ \\
\hline GPM-225 & $\mathbf{- 2 . 1 8} * *$ & $\mathbf{- 1 . 9 9} * *$ & 0.19 & -1.36 & 4.82 & 1.88 & $6.06 * *$ & $-1.99 * *$ & -0.21 \\
\hline GPM-255 & $-1.01 * *$ & $-1.66 * *$ & $\mathbf{- 0 . 6 4} * *$ & 0.48 & -8.68 & 0.75 & $-8.79 * *$ & $-1.66 * *$ & $0.59 * *$ \\
\hline GPM-628 & $-2.01 * *$ & $-1.66 * *$ & $0.36 *$ & $\mathbf{- 9 . 8 6} *$ & -7.18 & $-6.25 * *$ & $-9.27 * *$ & $-1.66 * *$ & $-0.46 *$ \\
\hline GPM-668 & $-0.84 *$ & $-1.49 * *$ & $\mathbf{- 0 . 6 4} * *$ & 0.14 & 2.99 & -1.98 & $\mathbf{1 2 . 9 0} * *$ & $-1.49 * *$ & 0.04 \\
\hline GPM-676 & $2.16 * *$ & $2.18 * *$ & 0.02 & 5.48 & 3.32 & 2.68 & $4.32 * *$ & $2.18 * *$ & 0.02 \\
\hline GPM-686 & -0.18 & 0.01 & 0.19 & -3.36 & -2.34 & $\mathbf{4 . 5 2} *$ & $-7.92 * *$ & 0.01 & 0.14 \\
\hline GPM-735 & $1.99 * *$ & $2.01 * *$ & 0.02 & 1.48 & 1.99 & -0.83 & 0.61 & $2.01 * *$ & 0.23 \\
\hline GPM-751 & $-\mathbf{2 . 1 8} * *$ & $-1.66 * *$ & $0.52 * *$ & -4.69 & -5.34 & 1.18 & $-2.91 * *$ & $-1.66 * *$ & $-0.35 *$ \\
\hline GPM-755 & $3.32 * *$ & $3.34 * *$ & 0.02 & -6.69 & $\mathbf{- 1 3 . 3 4} *$ & 1.78 & $-5.26 * *$ & $3.34 * *$ & 0.07 \\
\hline SE & 0.38 & 0.38 & 0.18 & 3.92 & 5.85 & 1.99 & 0.48 & 0.38 & 0.14 \\
\hline Testers & & & & & & & & & \\
\hline CML-451 & $0.80 * *$ & $0.70 * *$ & -0.10 & -2.47 & -0.20 & -1.20 & $-1.99 * *$ & $0.70 * *$ & $0.45 *$ \\
\hline CM-111 & $-0.80 * *$ & $-0.70 * *$ & 0.10 & 2.47 & 0.20 & 1.20 & $1.99 * *$ & $-0.70 * *$ & $-0.45 *$ \\
\hline SE & 0.14 & 0.14 & 0.07 & 1.43 & 2.14 & 0.73 & 0.17 & 0.14 & 0.05 \\
\hline
\end{tabular}

$*$ and $* *$ : Significance at $5 \%$ and $1 \%$ probability respectively 
Table.2b The GCA effects of parents for yield related traits

\begin{tabular}{|c|c|c|c|c|c|c|c|c|c|c|}
\hline $\begin{array}{c}\text { Parents } \\
\text { Lines }\end{array}$ & $\mathbf{C L}$ & $\mathbf{C G}$ & $\mathbf{K R N}$ & $\mathbf{K P R}$ & $\mathbf{C W}$ & $\mathbf{G W}$ & $\mathbf{S P}$ & $\mathbf{T W}$ & $\mathbf{F Y}$ & $\mathbf{G Y}$ \\
\hline GPM-04 & 0.94 & -0.46 & $-0.78 *$ & 2.72 & 18.74 & 15.29 & -1.53 & -0.58 & -0.36 & $-0.42 * *$ \\
\hline GPM-07 & -0.39 & -0.46 & -0.28 & -0.61 & $-30.76 *$ & $-22.04 *$ & 1.63 & $-4.41 * *$ & -0.02 & 0.07 \\
\hline GPM-26 & $\mathbf{2 . 4 4} * *$ & $0.71 *$ & 0.28 & 2.06 & $\mathbf{5 9 . 4 1} * *$ & $54.96 * *$ & $\mathbf{3 . 9 7} * *$ & $\mathbf{7 . 0 9} * *$ & $0.98 * *$ & $0.58 * *$ \\
\hline GPM-52 & -0.22 & 1.04 & -0.44 & -1.94 & -1.42 & -2.38 & -0.20 & 1.76 & $-0.52 *$ & $-0.65 * *$ \\
\hline GPM-114 & 0.28 & -0.62 & -0.28 & 0.89 & -7.4 & -4.38 & 0.07 & 2.59 & 0.1 & -0.2 \\
\hline GPM-119 & 0.06 & 0.54 & 0.06 & 1.72 & $26.74 *$ & $21.79 *$ & 0.63 & $3.09 * *$ & $1.02 * *$ & 0.17 \\
\hline GPM-225 & $1.56 *$ & 0.29 & -0.11 & -2.78 & -9.76 & -3.54 & 0.30 & -0.91 & -0.02 & $0.50 * *$ \\
\hline GPM-255 & $-1.56 *$ & -0.12 & 0.06 & 0.52 & -7.92 & -15.38 & -0.37 & 0.09 & $1.31 * *$ & -0.15 \\
\hline GPM-628 & $-2.22 * *$ & -0.46 & $\mathbf{0 . 8 9} * *$ & -1.94 & $-26.42 *$ & $-24.38 * *$ & $-3.03 *$ & -1.74 & $-0.52 *$ & 0.15 \\
\hline GPM-668 & -0.72 & 0.24 & 0.06 & -1.61 & $-27.59 *$ & $-22.04 *$ & -0.37 & -0.08 & -0.19 & 0.01 \\
\hline GPM-676 & 0.78 & -0.29 & $-0.78 *$ & 1.72 & -10.59 & -0.21 & -1.70 & $-2.24 *$ & 0.21 & $-0.65 * *$ \\
\hline GPM-686 & $\mathbf{2 . 4 4} * *$ & $\mathbf{1 . 0 4}$ & 0.11 & $\mathbf{3 . 7 2}$ & $50.24 * *$ & $40.29 * *$ & 0.97 & $2.59 *$ & $0.48 *$ & $\mathbf{0 . 7 1} * *$ \\
\hline GPM-735 & -0.89 & -0.12 & $\mathbf{0 . 8 9} * *$ & 0.22 & -15.09 & $-18.88 *$ & $-4.37 * *$ & -1.91 & $-0.86 * *$ & 0.01 \\
\hline GPM-751 & 0.14 & 0.04 & 0.22 & -0.94 & 12.41 & 1.12 & -0.70 & 0.76 & -0.02 & -0.15 \\
\hline GPM-755 & 0.28 & 0.38 & 0.32 & $-3.44 *$ & 21.01 & 9.79 & 1.30 & $-2.58 *$ & 0.31 & 0.08 \\
\hline SE & 0.64 & 0.35 & 0.31 & 1.41 & 11.88 & 9.25 & 1.19 & 1.11 & 0.22 & 0.15 \\
\hline Testers & & & & & & & & & \\
\hline CML-451 & 0.32 & 0.13 & $0.27 *$ & -0.98 & 7.12 & 4.83 & -0.44 & $1.06 *$ & -0.09 & $-0.15 * *$ \\
\hline CM-111 & -0.32 & -0.13 & $-0.27 *$ & 0.98 & -7.12 & -4.83 & 0.44 & $-1.06 *$ & 0.09 & $\mathbf{0 . 1 5} * *$ \\
\hline SE & 0.23 & 0.13 & 0.12 & 0.52 & 4.34 & 3.38 & 0.43 & 0.41 & 0.081 & 0.06 \\
\hline
\end{tabular}

$*$ and $* *$ : Significance at $5 \%$ and $1 \%$ probability respectively.

Graph 1a: Standard heterosis of better performing hybrids for morphophysiological traits

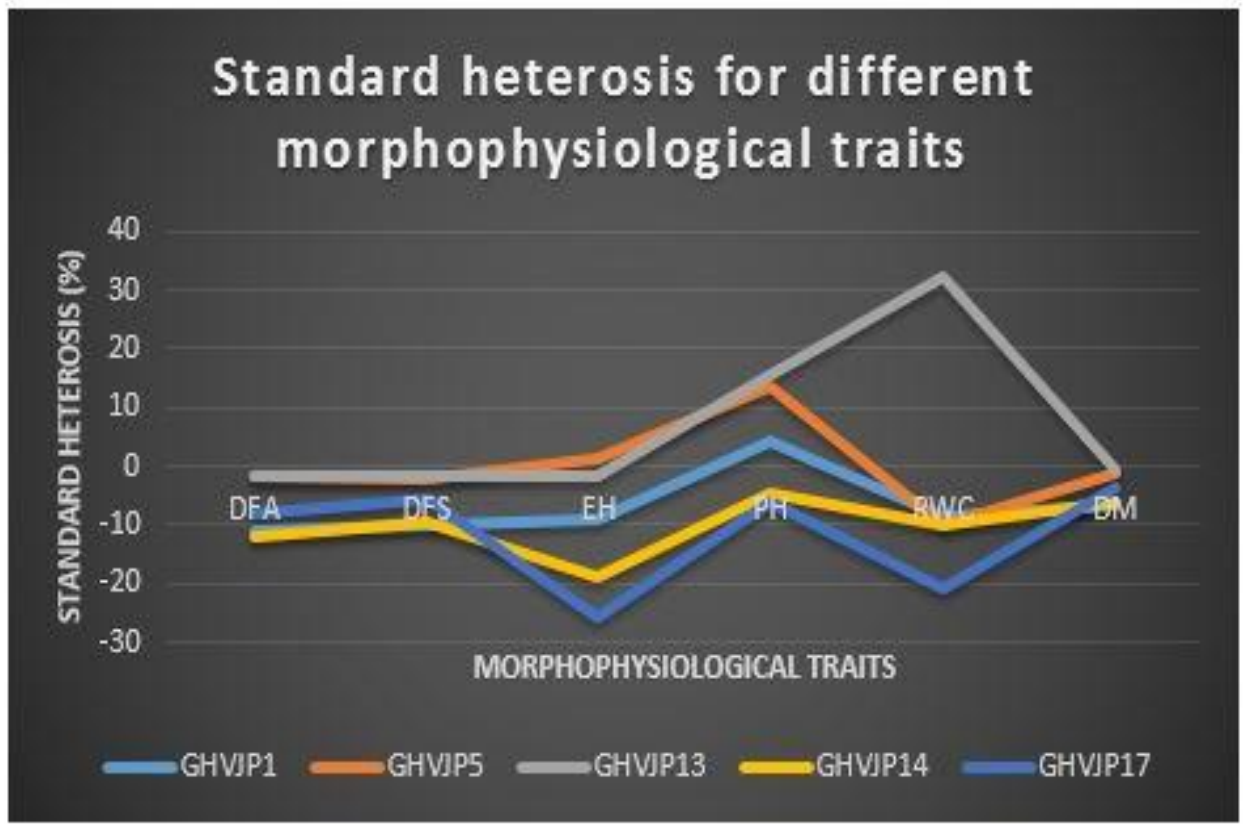


Table.3a The SCA effects of hybrids for morpho-physiological traits

\begin{tabular}{|c|c|c|c|c|c|c|c|c|}
\hline Hybrids & DFA & DFS & ASI & EH & PH & SPAD & RWC & DM \\
\hline GHVJP 1 & $-3.80 * *$ & $-3.70 * *$ & 0.10 & -1.20 & -3.47 & 4.47 & $-8.94 * *$ & $-3.70 * *$ \\
\hline GHVJP 2 & $3.80 * *$ & $3.70 * *$ & -0.10 & 1.20 & 3.47 & -4.47 & $8.94 * *$ & $3.70 * *$ \\
\hline GHVJP 3 & 0.70 & $1.13 *$ & 0.43 & -1.37 & -2.97 & -0.20 & -0.03 & $1.13 *$ \\
\hline GHVJP 4 & -0.70 & $-1.13 *$ & -0.43 & 1.37 & 2.97 & 0.20 & 0.03 & $-1.13 *$ \\
\hline GHVJP 5 & -0.97 & $-1.20 *$ & -0.23 & -0.20 & -0.80 & 2.70 & $-4.32 * *$ & $-1.20 *$ \\
\hline GHVJP 6 & 0.97 & $1.20 *$ & 0.23 & 0.20 & 0.80 & -2.70 & $4.32 * *$ & $1.20 *$ \\
\hline GHVJP 7 & $-1.13 *$ & -1.03 & 0.10 & 1.47 & 0.53 & -3.20 & $-3.01 * *$ & -1.03 \\
\hline GHVJP 8 & $1.13 *$ & 1.03 & -0.10 & -1.47 & -0.53 & 3.20 & $3.01 * *$ & 1.03 \\
\hline GHVJP 9 & -0.80 & -0.87 & -0.07 & -1.70 & -3.80 & -2.26 & $-4.21 * *$ & -0.87 \\
\hline GHVJP10 & 0.80 & 0.87 & 0.07 & 1.70 & 3.80 & 2.26 & $4.21 * *$ & 0.87 \\
\hline GHVJP11 & $1.53 * *$ & 0.97 & $-0.57 *$ & 5.97 & -1.13 & 4.77 & $14.81 * *$ & 0.97 \\
\hline GHVJP12 & $-1.53 * *$ & -0.97 & $0.57 *$ & -5.97 & 1.13 & -4.77 & $-14.81 * *$ & -0.97 \\
\hline GHVJP13 & $2.53 * *$ & $1.97 * *$ & $-0.57 *$ & 10.13 & 15.53 & 0.59 & $15.78 * *$ & $1.97 * *$ \\
\hline GHVJP14 & $-2.53 * *$ & $-1.97 * *$ & $0.57 *$ & -10.13 & -15.53 & -0.59 & $-15.78 * *$ & $-1.97 * *$ \\
\hline GHVJP15 & $1.70 * *$ & $1.63 * *$ & -0.07 & 2.63 & 1.03 & 0.19 & $5.80 * *$ & $1.63 * *$ \\
\hline GHVJP16 & $-1.70 * *$ & $-1.63 * *$ & 0.07 & -2.63 & -1.03 & -0.19 & $-5.80 * *$ & $-1.63 * *$ \\
\hline GHVJP17 & $-1.63 * *$ & -1.03 & $0.60 *$ & -2.70 & -5.80 & -2.75 & $-3.23 * *$ & -1.03 \\
\hline GHVJP18 & $1.63 * *$ & 1.03 & $-0.60 *$ & 2.70 & 5.80 & 2.75 & $3.23 * *$ & 1.03 \\
\hline GHVJP19 & -0.13 & -0.20 & -0.07 & 1.30 & -5.97 & -3.38 & $-4.71 * *$ & -0.20 \\
\hline GHVJP20 & 0.13 & 0.20 & 0.07 & -1.30 & 5.97 & 3.38 & $4.71 * *$ & 0.20 \\
\hline GHVJP21 & $3.20 * *$ & $3.13 * *$ & -0.07 & -0.70 & 4.37 & -0.31 & $3.39 * *$ & $3.13^{* *}$ \\
\hline GHVJP22 & $-3.20 * *$ & $-3.13 * *$ & 0.07 & 0.70 & -4.37 & 0.31 & $-3.39 * *$ & $-3.13 * *$ \\
\hline GHVJP23 & $-1.80 * *$ & $-1.70 * *$ & 0.10 & -1.87 & -2.97 & 2.19 & $-10.63 * *$ & $-1.70 * *$ \\
\hline GHVJP24 & $1.80 * *$ & $1.70 * *$ & -0.10 & 1.87 & 2.97 & -2.19 & $10.63 * *$ & $1.70 * *$ \\
\hline GHVJP25 & -0.30 & -0.37 & -0.07 & -0.03 & 9.37 & -1.83 & $-12.11 * *$ & -0.37 \\
\hline GHVJP26 & 0.30 & 0.37 & 0.07 & 0.03 & -9.37 & 1.83 & $12.11 * *$ & 0.37 \\
\hline GHVJP27 & $1.20 *$ & $1.63 * *$ & 0.43 & -7.53 & 1.03 & -1.25 & $10.67 * *$ & $1.63 * *$ \\
\hline GHVJP28 & $-1.20 *$ & $-1.63 * *$ & -0.43 & 7.53 & -1.03 & 1.25 & $-10.67 * *$ & $-1.63 * *$ \\
\hline GHVJP29 & -0.30 & -0.37 & -0.07 & -4.20 & -4.97 & 0.25 & 0.76 & -0.37 \\
\hline GHVJP30 & 0.30 & 0.37 & 0.07 & 4.20 & 4.97 & -0.25 & -0.76 & 0.37 \\
\hline SE & 0.53 & 0.54 & 0.25 & 5.54 & 8.27 & 2.82 & 0.68 & 0.54 \\
\hline
\end{tabular}

$*$ and **: Significance at $5 \%$ and $1 \%$ probability respectively. 
Table.3b The SCA effects of hybrids for yield related traits

\begin{tabular}{|c|c|c|c|c|c|c|c|c|c|c|}
\hline Hybrids & CL & CG & KRN & KPR & CW & GW & SP & TW & FY & GY \\
\hline GHVJP 1 & 0.01 & 0.03 & -0.27 & -0.86 & -3.46 & -3.67 & -0.56 & 0.78 & $-1.91 * *$ & -0.02 \\
\hline GHVJP 2 & -0.01 & -0.03 & 0.27 & 0.86 & 3.46 & 3.67 & 0.56 & -0.78 & $1.91 * *$ & 0.02 \\
\hline GHVJP 3 & 0.34 & 0.70 & 0.07 & -1.19 & -5.29 & -4.67 & -0.39 & -0.39 & 0.09 & 0.06 \\
\hline GHVJP 4 & -0.34 & -0.70 & -0.07 & 1.19 & 5.29 & 4.67 & 0.39 & 0.39 & -0.09 & -0.06 \\
\hline GHVJP 5 & $1.82 *$ & 0.53 & -0.10 & $4.81 *$ & $35.54 *$ & $30.67 *$ & -0.06 & $4.44 *$ & $0.76^{*}$ & $0.88 * *$ \\
\hline GHVJP 6 & $-1.82^{*}$ & -0.53 & 0.10 & $-4.81 *$ & $-35.54 *$ & $-30.67 *$ & 0.06 & $-4.44^{*}$ & $-0.76 *$ & $-0.88 * *$ \\
\hline GHVJP 7 & -0.49 & -0.13 & $-0.93 *$ & 1.48 & -11.96 & -10.67 & -0.56 & -0.56 & -0.41 & -0.38 \\
\hline GHVJP 8 & 0.49 & 0.13 & $0.93 *$ & -1.48 & 11.96 & 10.67 & 0.56 & 0.56 & 0.41 & 0.38 \\
\hline GHVJP9 & -0.32 & -0.47 & -0.10 & 0.31 & 6.04 & 3.67 & -0.56 & 1.11 & -0.08 & -0.21 \\
\hline GHVJP10 & 0.32 & 0.47 & 0.10 & -0.31 & -6.04 & -3.67 & 0.56 & -1.11 & 0.08 & 0.21 \\
\hline GHVJP11 & 1.01 & 0.7 & 0.23 & $-4.52 *$ & $37.88 *$ & $33.83 *$ & 1.94 & 1.44 & 0.42 & $-0.68 *$ \\
\hline GHVJP12 & -1.01 & -0.7 & -0.23 & $4.52 *$ & $-37.88 *$ & $-33.83 *$ & -1.94 & -1.44 & -0.42 & $0.68 *$ \\
\hline GHVJP13 & -0.49 & -0.8 & $1.23 * *$ & -2.02 & -12.96 & -8.83 & 0.61 & -0.22 & $0.76 *$ & $0.50 *$ \\
\hline GHVJP14 & 0.49 & 0.8 & $-1.23 * *$ & 2.02 & 12.96 & 8.83 & -0.61 & 0.22 & $-0.76 *$ & $-0.50 *$ \\
\hline GHVJP15 & -0.49 & -0.63 & -0.77 & -0.36 & -21.46 & -14.00 & 1.61 & -0.56 & -0.58 & 0.12 \\
\hline GHVJP16 & 0.49 & 0.63 & 0.77 & 0.36 & 21.46 & 14.00 & -1.61 & 0.56 & 0.58 & -0.12 \\
\hline GHVJP17 & 0.18 & 0.70 & 0.40 & -0.19 & 11.71 & 11.33 & 1.94 & -1.39 & $0.92 * *$ & 0.22 \\
\hline GHVJP18 & -0.18 & -0.70 & -0.40 & 0.19 & -11.71 & -11.33 & -1.94 & 1.39 & $-0.92 * *$ & -0.22 \\
\hline GHVJP19 & -0.66 & -0.13 & 0.07 & -1.19 & -14.12 & -12.33 & -0.72 & -2.06 & $0.92 * *$ & -0.05 \\
\hline GHVJP20 & 0.66 & 0.13 & -0.07 & 1.19 & 14.12 & 12.33 & 0.72 & 2.06 & $-0.92 * *$ & 0.05 \\
\hline GHVJP21 & -0.84 & $-1.13^{*}$ & -0.27 & -1.19 & -10.71 & -4.67 & -2.06 & -0.56 & $-0.91 * *$ & -0.38 \\
\hline GHVJP22 & 0.84 & $1.13^{*}$ & 0.27 & 1.19 & 10.71 & 4.67 & 2.06 & 0.56 & $0.91 * *$ & 0.38 \\
\hline GHVJP23 & $1.84 *$ & -0.13 & 0.07 & $5.48 *$ & 43.79* & $37.17 * *$ & -1.39 & -0.39 & -0.41 & $0.72 *$ \\
\hline GHVJP24 & $-1.84 *$ & 0.13 & -0.07 & $-5.48 *$ & $-43.79 *$ & $-37.17 * *$ & 1.39 & 0.39 & 0.41 & $-0.72 *$ \\
\hline GHVJP25 & -0.16 & 0.37 & -0.60 & 2.31 & 0.38 & -0.83 & -0.39 & -0.22 & -0.08 & $0.45 *$ \\
\hline GHVJP26 & 0.16 & -0.37 & 0.60 & -2.31 & -0.38 & 0.83 & 0.39 & 0.22 & 0.08 & $-0.45^{*}$ \\
\hline GHVJP27 & 0.18 & 0.20 & $1.07 *$ & -0.52 & 14.88 & 14.83 & 1.61 & -0.22 & 0.09 & 0.25 \\
\hline GHVJP28 & -0.18 & -0.20 & $-1.07 *$ & 0.52 & -14.88 & -14.83 & -1.61 & 0.22 & -0.09 & -0.25 \\
\hline GHVJP29 & 0.01 & 0.20 & -0.10 & -1.36 & -0.12 & -1.83 & -1.06 & 0.78 & 0.42 & -0.28 \\
\hline GHVJP30 & -0.01 & -0.20 & 0.10 & 1.36 & -0.12 & 1.83 & 1.06 & -0.78 & -0.42 & 0.28 \\
\hline SE & 0.90 & 0.49 & 0.45 & 1.99 & 16.80 & 13.09 & 1.68 & 1.58 & 0.31 & 0.22 \\
\hline
\end{tabular}

$*$ and **: Significance at $5 \%$ and $1 \%$ probability respectively 
Table.4 Yield traits of good performing hybrids

\begin{tabular}{|c|c|c|c|c|c|c|c|c|c|}
\hline Pedigree & Hybrid & $\begin{array}{c}\text { CL } \\
(\mathbf{c m})\end{array}$ & $\begin{array}{c}\text { CG } \\
(\mathbf{c m})\end{array}$ & KRN & KPR & $\begin{array}{c}\text { CW } \\
(\mathbf{g})\end{array}$ & $\begin{array}{c}\text { GW } \\
\mathbf{( g )}\end{array}$ & $\begin{array}{c}\text { TW } \\
(\mathbf{g})\end{array}$ & $\begin{array}{c}\text { GY } \\
(\mathbf{k g} / \mathbf{h a})\end{array}$ \\
\hline GPM26 x CML451 & GHVJP-5 & 21.33 & $\mathbf{1 6 . 0 0}$ & 14.00 & 37.00 & $\mathbf{2 7 1 . 6 7}$ & $\mathbf{2 2 0 . 3 3}$ & $\mathbf{3 7 . 0 0}$ & 10.83 \\
\hline GPM686 x CML451 & GHVJP-23 & $\mathbf{2 2 . 3 3}$ & 15.77 & 14.43 & $\mathbf{3 9 . 3 3}$ & 241.67 & 184.67 & 29.67 & 10.36 \\
\hline GPM119 x CM111 & GHVJP-12 & 16.33 & 14.33 & 13.67 & 37.33 & 155.33 & 118.00 & 27.00 & 10.19 \\
\hline GPM225 x CML 451 & GHVJP-13 & 16.00 & 13.67 & $\mathbf{1 5 . 3 3}$ & 27.33 & 148.00 & 120.33 & 26.33 & 10.09 \\
\hline GPM735 x CML451 & GHVJP-25 & 17.00 & 15.00 & 14.67 & 34.67 & 166.00 & 117.00 & 25.33 & 9.75 \\
\hline CHECK & NK6240 (NC) & 17.70 & 15.83 & 14.40 & 31.20 & 181.13 & 138.83 & 28.67 & 8.06 \\
\hline & GPMH1101 (LC) & 16.47 & 15.00 & 13.47 & 31.47 & 181.87 & 132.43 & 25.33 & 8.50 \\
\hline CV (\%) & & 8.42 & 5.10 & 5.44 & 10.71 & 16.30 & 16.48 & 9.873 & 12.56 \\
\hline CD at 5\% & & 2.43 & 1.22 & 1.25 & 5.75 & 46.46 & 36.38 & 4.227 & 1.79 \\
\hline CD at 1\% & & 3.22 & 1.62 & 1.66 & NS & 61.66 & 48.29 & 5.611 & 2.38 \\
\hline
\end{tabular}

Graph 1b: Standard heterosis of better performing hybrids for yield traits.

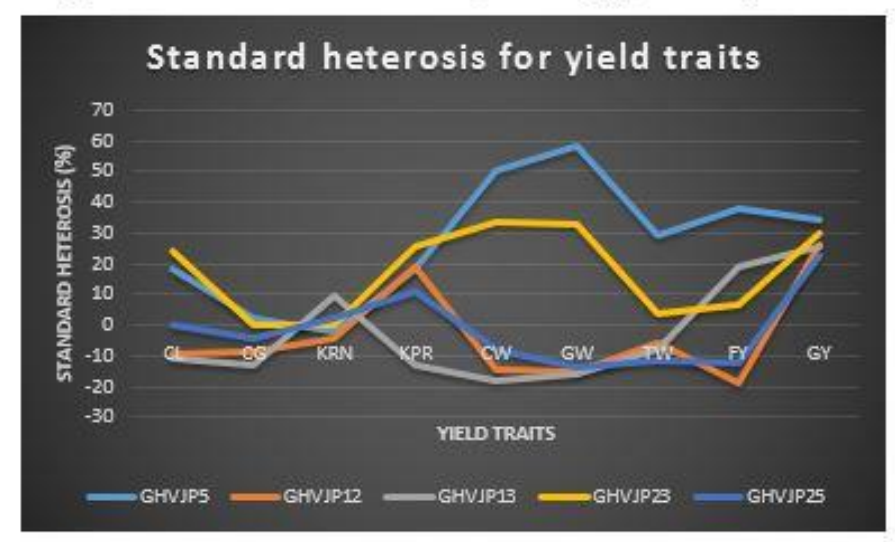

The superior crosses that were the result of good $\times$ poor general combiners may be due to the interaction between the positive alleles from good combiners and negative alleles from poor combiners as parents which under heterozygous condition resulted in high heterosis due to masking effect of favourable alleles at different loci. The high yield of such crosses would be non-fixable and thus could be exploited for heterosis breeding. The case of high SCA between good $\times$ poor combiners could produce good segregants only if the additive genetic effects are present in the good general combiners and complementary epistatic effects in the poor combiners and they act in the same direction to maximize desirable plant attributes.
In the present study it was found that the variance due to SCA was more than the variance due to GCA indicating the predominance of non-additive gene action which arises largely due to dominance and epistatic interactions (Sandesh et al., 2018) and selection for such traits can be performed using recurrent selection for GCA method.

\section{Standard Heterosis}

In the present investigation standard check (NK6240) was taken into consideration for estimating heterosis as it is economically more beneficial than heterobeltiosis and mid parent heterosis. SCA is the indicative of heterosis for the evaluation of hybrids. The 
SCA is due to non-additive gene interactions and the extent of heterosis depends on the magnitude of non-additive gene action and wide genetic diversity among parents.

For early maturity, the hybrids GHVJP1 (GPM-04×CML-451) and GHVJP14 (GPM$225 \times \mathrm{CM}-111)$ showed significant negative heterosis with respect to DFA, DFS and DM. The hybrid GHVJP13 (GPM-225×CML-451) showed significant positive heterosis for $\mathrm{PH}$ as well as RWC (Graph 1a). It also had high SCA and per se values for yield. Hence can be considered as drought tolerant and subjected to further evaluation.

In case of yield traits, the hybrids GHVJP5 (GPM 26×CML 451) and GHVJP23 (GPM $686 \times$ CML 451) showed significant positive heterosis for CL, KPR, CW, GW, TW and GY. The hybrid GHVJP12 (GPM119×CM 111) showed significant positive heterosis for KPR and GY. The hybrid GHVJP13 (GPM$225 \times$ CML-451) showed significant positive heterosis for KRN, FY and GY (Graph 1b).

From all these observations it can be said that the hybrids GHVJP5, GHVJP23, GHVJP12, GHVJP 13 and GHVJP 25 were better performing with respect to yield and yield related traits (Table 4). These hybrids can be further evaluated in large scale and can be used for the exploitation of standard heterosis. Similar findings were obtained by Wali et al., (2010).

In the present investigation, it was found that the percent contribution of lines was much greater than testers for most of the studied traits indicating that the female parent contributed maximum for total variance in hybrids followed by interaction. This is in conformity with Amin et al., (2014).

Any combination among parents may produce hybrid vigour over parents which might be due to dominant, over dominant or epistatic gene action (Moll and Stuber 1974). So, the crosses showing desirable SCA effects can be used in future breeding programmes for exploiting herterosis (GHVJP-5, GHVJP-23, GHVJP-12, GHVJP-13 and GHVJP-25). The inbred lines selected for high GCA can be used for the development of synthetic varieties or population to derive inbred lines. In the present investigation, the promising crosses were the result of good $\mathrm{x}$ good (GHVJP-5, GHVJP-23), good $\mathrm{x}$ poor (GHVJP-12) and poor x good (GHVJP13, GHVJP-25) general combiners as parents. The result, therefore, revealed that high gca value of a parent is no guarantee of high sca effect of their crosses and conforming the earlier reports of Dubey et al., (2001). It was also be found in some hybrids that the hybrids with high per se performance need not always reveal high SCA effect and vice versa (Peremlatha and Kalmani 2010). Hence selection must be made for such hybrids which show high per se values along with significant SCA effects for concerned traits.

\section{Abbreviations}

DFA: Days to fifty percent anthesis

DFS: Days to fifty percent silking

ASI: Anthesis silking interval

EH: Ear height $(\mathrm{cm})$

PH: Plant height $(\mathrm{cm})$

SPAD: SPAD meter reading (relative chlorophyll content)

RWC: Relative water content (percent)

DM: Days to maturity

TLB: Turcicum Leaf Blight

MLB: Maydis Leaf Blight

CL: Cob length $(\mathrm{cm})$

CG: Cob girth $(\mathrm{cm})$

KRN: Kernel row number

KPR: Kernel per row

CW: Cob weight (g)

GW: Grain weight (g)

SP: Shelling percentage (percent) 
TW: Test weight $(\mathrm{g})$

FY: Fodder yield ( $\mathrm{t} / \mathrm{ha})$

GY: Grain yield (t/ha)

GCA: General combining ability

SCA: Specific combining ability

\section{References}

Allard RW, 1960. Principles of plant breeding. John \& sons. Inc. New York and London.

Amin AE, Ahmad SA, Shouny KA, Olfat HE, Ibrahim KIM, 2014. Heterosis and combining ability in yellow maize (Zea mays, L.) crosses under two planting dates. Annals Agric. Sci. Cairo 49(2): 531-543.

Anonnymous. 2018. World agricultural production, United State Department of Agriculture: 15.

Anonymous. 2017. State wise area, production and productivity of Maize in India. https://www.indiaagristat.com.

Dubey RB, Joshi VN, Pandiya NK, 2001. Heterosis and combining ability for quality, yield and maturity traits in conventional and non-conventional hybrids of maize. Indian J. Genet 61(4): 353-355.

Ejigu YG, Tongoona PB, and Ifie BE, 2017. General and specific combining ability studies of selected tropical white maize inbred lines for yield and yield related traits. Int. J. Agrl. Sci. and Res 7(2):381-396.

Izhar T, Chakraborty M, 2013. Combining ability and heterosis for grain yield and its components in maize inbreds over environments (Zea mays L.). African J. Agrl. Res 8(25): 3276-3280.

Kempthorne O, 1957. An introduction to genetic statistics. John Wiley and Sons Inc N. Y: 456-471.

Moll RH, Stuber CW, 1974. Quantitative genetics empirical results relevant to plant breeding. Ad. Agron. J 26: 277313.

Panda S, Wali MC, Kachapur RM, Harlapur SI, 2017. Combining Ability and Heterosis Analysis of Single Cross Hybrids of Maize (Zea mays L.). Int. J. Curr. Microbiol. App. Sci 6(10): 2608-2618.

Payak MM, Sharma RC, 1983. Disease rating scales in maize in India. Techniques of scoring for resistance to important diseases of maize. All India Coordinated Maize Improvement Project, Indian Agricultural Research Institute, New Delhi: $1-4$

Premlatha M, Kalamani A, 2010. Heterosis and combining ability studies in maize (Zea mays L.). Int. J. Pl. Sci 5(1): 369-372.

Sandesh GM, Karthikeyan A, Kavithamani D, Thangaraj K, Ganesan KN, Ravikesavan R, Senthil N, 2018. Heterosis and combining ability studies for yield and its component traits in Maize (Zea mays L.). Electronic J. Pl. Breed 9(3): 10121023.

Wali MC, Kachapur RM, Chandrashekhar CP, Kulkarni VR, Devaranavadagi SB, 2010. Gene action and combining ability studies in single cross hybrids of maize (Zea mays L.). Karnataka J. Agric. Sci 23(4): 557-562.

\section{How to cite this article:}

Meghashri S. Patil, B. N. Motagi and Kachapur, R. M. 2020. Heterosis and Combining Ability Studies in Maize (Zea mays L.) for Drought Tolerance, TLB Disease Resistance and Productivity in Northern Dry Tract of Karnataka. Int.J.Curr.Microbiol.App.Sci. 9(10): 10541064. doi: https://doi.org/10.20546/ijcmas.2020.910.126 